Rev Soc Esp Dolor

2013; 20(6): 332-333

\section{Hacia la perspectiva GRADE}

\section{Sr. Director:}

Esta última década se ha caracterizado por un incremento enorme y exponencial de la cantidad de información biomédica publicada lo cual puede ser beneficioso o llegar a convertirse en un gran problema para los clínicos.

Es por esto que surge una nueva corriente denominada "medicina basada en evidencia", la cual nace como un movimiento destinado a llenar la tradicional brecha existente entre la "práctica clínica y la investigación", promoviendo el cambio desde la toma de decisiones clínicas "informadas" para ser aplicadas en un paciente "particular".

Es por esto que hoy en día cada vez tiene más peso entre los profesionales de la salud tomar una decisión clínica fundamentada en los mejores niveles de evidencia; esto quiere decir "hasta qué punto los clínicos pueden confiar en que la estimación de un efecto es la adecuada para apoyar una recomendación"; esto generalmente está asociado con el diseño del estudio (descriptivos o analíticos, observacionales o experimentales). También debemos recordar que cada diseño presenta diferentes características y que "no" todas las preguntas clínicas se deben responden con un mismo diseño metodológico. La fuerza de recomendación nos indica hasta qué punto podemos confiar si poner en práctica la recomendación nos llevará a producir más beneficios que riesgos.

En la toma de decisión clínica, existen conceptos relevantes, como es el caso de:

- Calidad (nivel) de la evidencia y fuerza (grado) de recomendación: ambos constituyen un pilar fundamental de la práctica basada en evidencia, en su intento de "estandarizar" y proporcionar a los clínicos reglas para analizar los artículos científicos, determinar su validez y considerar su utilidad clínica (balance entre los riesgos versus beneficios).

Dentro de los elementos "relevantes" que debemos considerar al momento de tomar una decisión clínica, y así poder "recomendar" con "seguridad" la utilización de una intervención, es poner en una balanza los "posibles beneficios" versus "posibles riesgos"; este punto es clave debido a que considera no solo los posibles efectos adversos que pudiera presentar la intervención, sino que también incluye la magnitud del efecto, daño, coste, los valores y preferencias de los pacientes.

El primer intento confiable para introducir "rigor" y "transparencia" en la jerarquización de la evidencia fue realizado hace más de 30 años por la Canadian Task Force on Preventive Health Care (CTFPHC) (1), adaptado posteriormente por la United State Preventive Services Task Force (USPSTF) (2). Desde entonces se han creado numerosas organizaciones e instituciones entre las que destacan el Centre for Evidence-Based Medicine (CEBM) de la Oxford (3), el Scottish Intercollegiate Guidelines Network (SIGN) (3), el National Institute for Health and Clinical Excellence (NICE) (4), Van Tulder (4) y, actualmente, Grade Working Group (GRADE) (5). Cada una de las instituciones mencionadas ha ido desarrollando sus propios sistemas jerárquicos. Actualmente podemos contabilizar más de cien herramientas, 19 sistemas para evaluar la calidad y 7 para graduar las recomendaciones (5).

En el año 2000, con el objetivo de abordar las deficiencias de los actuales sistemas de clasificación, un grupo internacional de clínicos y en su mayoría metodólogos crearon la iniciativa GRADE (The Grading of Recommendations Assessment Development and Evaluation/ Clasificación de la Evaluación, Desarrollo y Valoración de las Recomendaciones), quienes han elaborado una clasificación que presenta un enfoque sistemático y explícito, a partir de la definición de una serie de criterios necesarios para hacer juicios sobre la calidad de la evidencia y fuerza de la recomendación.

Actualmente el grupo de trabajo GRADE está compuesto por 161 profesionales del área de la salud y metodólogos distribuidos por todo el mundo (para mayor información visitar http://www.gradeworkinggroup.org/ about_us.htm).

Hoy en día un gran número de instituciones utilizan y apoyan la perspectiva del grupo de trabajo GRADE, entre 
ellas encontramos a la Organización Mundial de la Salud (OMS), la Colaboración Cochrane, el British Medical Journal (BMJ) y Clinical Evidence, entre otras (para mayor información sobre las instituciones que apoyan o utilizan la metodología GRADE, visitar http://www.gradeworkinggroup.org/society/index.htm).

R. Aguilera Eguía ${ }^{1}$, E. Zafra Santos², G. Pavez Von Martens $^{2}$, C. Rojas Sepúlveda ${ }^{3}$, G. Aguayo Alcayala ${ }^{3}$, D. Sánchez León ${ }^{3}$, C. Cofre Bolados ${ }^{2}$ y A. Espinoza Salinas ${ }^{2}$

\begin{abstract}
${ }^{1}$ Musculoskeletal Group Cochrane Colaboration; Member of the Grade Working Group; Member of the Cochrane Trainer's Network, Colaboración Cochrane Iberoamericana, Red Chilena de Salud Basada en Evidencia. Universidad Santiago de Chile. ${ }^{2}$ Escuela de Kinesiología. Universidad Santo Tomas. Santiago de Chile.

${ }^{3}$ Licenciado en Kinesiología. Santiago de Chile (Raúl Aguilera Eguía: kine.rae@gmail.com)
\end{abstract}

\section{Bibliografía}

1. Holger JS, Best D, Vist G, Andrew DO, for the GRADE working group. Letters, numbers, symbols and words: How to communicate grades of evidence and recommendations. CMAJ 2003; 169:677-80.

2. Atkins D, Best D, Briss PA, Eccles M, Falck-ytter Y, Flottorp S, et al.; Grade Working Group. Grading quality of evidence and strength of recommendations. BMJ 2004;328:1490-4.

3. Van Tulder M, Furlan A, Bombardier C, Bouter L, Editorial Board of the Cochrane Collaboration Back Review Group. Updated method guidelines for systematic reviews in the Cochrane collaboration back review group. Spine 2003;28:1290-9.

4. Martín Muñoz P, González de Dios J. valoración de la calidad de evidencia y fuerza de recomendaciones (I). Evapediatr 2010;6:63.

5. Martín Muñoz P, González de Dios J. Valoración de la calidad de evidencia y fuerza de recomendaciones (II). Consideraciones prácticas en la aplicación del sistema GRADE. Evapediatr 2010;6:91.

\section{Curso de Actualización en Dolor de la SED 2013}

\section{Sr. Director:}

Uno de los objetivos de la SED, y por tanto de su revista, ha sido ofrecer al socio y al lector instrumentos para la formación continuada en dolor que contaran con el aval y la acreditación de los organismos correspondientes. Durante los pasados años, la revista ha estado acreditada en su totalidad y de sus artículos se han extraído preguntas para los exámenes test que permitían obtener la acreditación.

La acreditación nos la otorgó la SEAFORMEC, que recientemente ha cesado en esta función, la cual ha sido asumida por el Ministerio de Sanidad. Se nos ha comunicado un cambio en los procedimientos de acreditación para las revistas médicas de tal modo que ya no se acreditan revistas sino artículos.

Para dar respuesta a las solicitudes de formación continuada de los socios de la SED hemos diseñado un curso de formación continuada "on line", habiéndose solicitado por esta nueva vía la Acreditación a la Comisión de Formación Continuada de las Profesiones Sanitarias. Será un curso de "Actualización en dolor de la SED", con una periodicidad anual. El material de estudio procederá de artículos originales, revisiones, medicina basada en la evidencia y artículos relevantes de la Revista de la Sociedad Española del Dolor.

La sistemática será la misma que tuvimos en las pasadas ediciones de la formación continuada de la revista de la SED; si bien, hemos adaptado la plataforma de formación continuada para que el material de estudio esté colgado en dicha plataforma y sea de fácil acceso. Habrá que superar un examen test para poder obtener el certificado del curso.

Este curso será para los socios de la SED y sin costes. El número de alumnos admitidos será limitado y por orden de inscripción.

V. Mayoral Rojals, L. M. Torres Morera, J. L. Ortega García y F. Neira Reina

Directores Docentes del Curso de "Actualización en Dolor de la SED 2013" (Victor Mayoral Rojals: victormayoral@mac.com) 